\title{
Microalbuminuria and the Combination of MRI Markers of Cerebral Small Vessel Disease
}

\author{
Andrea Vilar-Bergua ${ }^{a} \quad$ Iolanda Riba-Llena ${ }^{a}$ Natalia Ramos ${ }^{b}$ Xavier Mundet ${ }^{c}$ \\ Eugenia Espinel $^{\mathrm{b}}$ Antonio López-Rueda ${ }^{\mathrm{a}}$ Elena Ostos $^{\mathrm{a}}$ Daniel Seron $^{\mathrm{b}}$ \\ Joan Montaner ${ }^{a}$ d Pilar Delgado ${ }^{a}$ \\ ${ }^{a}$ Neurovascular Research Laboratory, Vall d'Hebron Research Institute and bepartment of Nephrology, \\ Hypertension Unit, Departament de Medicina, Universitat Autònoma de Barcelona, ' Barcelona City Research \\ Support Unit-IDIAP Jordi Gol, Universitat Autònoma de Barcelona, and d'Stroke Unit, Neurology Department, \\ Vall d'Hebron Hospital, Barcelona, Spain
}

\section{Key Words}

Cerebrovascular diseases · White matter hyperintensities ·

Lacunar infarcts · Renal disease

\begin{abstract}
Background: Kidney function has been related to the presence of individual markers of cerebral small vessel disease (CSVD), as lacunes, white matter hyperintensities (WMH) or microbleeds. We aimed at studying the relationship of kidney dysfunction with the combination of several markers of CSVD. Methods: Subjects are those included in the ISSYS COhort (Investigating Silent Strokes in hypertensives: a magnetic resonance imaging study). A scale ranging from 0 to 4 points was applied based on the presence (one point each) of lacunes, deep microbleeds, moderate to extensive basal ganglia enlarged perivascular spaces (EPVS), and periventricular or deep WMH. We determined the creatinine-based glomerular filtration rate and the urinary albumin-to-creatinine ratio (UACR) as markers of kidney function and studied their association with the scale of CSVD in univariate and ordinal logistic regression analyses. Results: Among the 975
\end{abstract}

\section{KARGER}

E-Mail karger@karger.com

www.karger.com/ced patients included, $28.2 \%$ presented one or more CSVD markers, being the most prevalent marker (either alone or in combination) basal ganglia EPVS. The UACR was elevated at increasing the scores of the CSVD scale and remained as independent predictor of the combination of markers (common OR per natural log unit increase in UACR: 1.23, 1.07-1.41) after controlling per age, gender, cardiovascular risk, antihypertensive treatment and hypertension duration. In contrast, no associations were found between the CSVD scores and the creatinine-based estimated glomerular filtration rate. Conclusions: A significant proportion of stroke-free hypertensives present at least one imaging marker of CSVD. UACR but not creatinine-based glomerular filtration rate is associated with the combination of markers of CSVD.

(c) 2016 S. Karger AG, Basel

\section{Introduction}

Hypertension (HTN) is one of the most relevant modifiable risk factors of cerebral small vessel disease (CSVD), a term used to describe a set of clinical, cogni-
(C) 2016 S. Karger AG, Basel

1015-9770/16/0422-0066\$39.50/0
Dr. Pilar Delgado

Neurovascular Research Laboratory, Vall d'Hebron Research Institute (VHIR) Hospital Vall d'Hebron, Passeig Vall d'Hebron 119-129

ES-08035 Barcelona (Spain)

E-Mail pilar.delgado@vhir.org 
tive, neuroimaging and neuropathological findings that emerge from disease affecting the perforating cerebral arterioles, capillaries and venules in the brain [1]. Neuroimaging manifestations of CSVD reflect damage in the white and deep grey matter and include acute lacunar (or small subcortical) infarcts or haemorrhages, lacunes (fluid-filled cavities thought to reflect old infarcts), white matter hyperintensities (WMH), brain atrophy and enlarged perivascular spaces (EPVS) [2]. Previous studies have shown associations between the renal function and the presence of these markers of CSVD in population-based studies and hypertensive cohorts, involving different ethnic groups [3-9]. Although there are some inconsistencies, overall, they suggest that impaired kidney function could be a risk factor for CSVD.

Nevertheless, while kidney function has been related with the presence of different individual small vessel disease markers, whether renal function also relates to their combination has been less studied. This might be important, considering that these imaging markers of CSVD often occur simultaneously within the same individuals. In this sense, in 2013, a simple scale that combines the presence of 4 different imaging markers of CSVD (lacunes, periventricular or deep WMH, deep microbleeds and basal ganglia EPVS) was described in hypertensives and subjects with first-ever lacunar stroke $[10,11]$. This scale recognizes that different CSVD markers of arteriolosclerotic origin tend to coexist or accumulate over time within subjects and allows the study of their combination rather than focusing in only one specific marker.

Also, this scale combines ischemic (i.e. lacunes, WMH) and haemorrhagic manifestations of CSVD and after its first description, has been further validated in 2 independent cohorts. Staals et al. [12] found the score related to lacunar strokes as compared to cortical strokes, suggesting that it is actually a measure of CSVD, and more recently, Xiao et al. [13] applied this scale to a cohort of individuals with first-ever lacunar stroke, where the increasing number of CSVD markers was found associated with worsening kidney function. To our knowledge, no previous studies have described an association between kidney function and the combination of several different CSVD markers in hypertensive individuals with no prior stroke.

This study aimed at measuring the combination of different CSVD markers by applying a predefined scale in a large cohort of asymptomatic hypertensive subjects and to investigate its relationship with kidney function parameters.

Microalbuminuria and CSVD

\section{Methods}

\section{Study Population}

This study was conducted within the ISSYS cohort (Investigating Silent Strokes in hypertensives: a magnetic resonance imaging study). Our detailed study protocol has been published elsewhere [14]. Briefly, this study included randomly selected individuals diagnosed with primary HTN routinely treated by general practitioners in an urban area of Barcelona that has Vall d'Hebron Hospital as reference centre. Inclusion criteria comprised (a) age between 50 and 70 years at the time of inclusion; (b) primary HTN diagnosed at least 1 year earlier; (c) no previous history of stroke or dementia (ascertained by clinical records and interview with the patients); (d) able to undergo a brain MRI. We enrolled 1,037 subjects in this study and from them, $976(94.1 \%)$ completed all baseline procedures, including a brain MRI scan.

This study was approved by our local institutional review Ethics Committee, and all subjects gave their informed consent to participate prior to the inclusion.

\section{Clinical Covariates and Kidney Function Parameters}

On the baseline visit, data on demographics and clinical history were collected. Apart from traditional vascular risk factors (diabetes, dyslipidaemia, current smoking habit, alcohol abuse and obesity), we calculated the global cardiovascular risk (which is the 10 -year risk of having a coronary event) by applying the Framingham-calibrated Registre Gironí del Cor (REGICOR) equation. According to REGICOR, participants are classified as low risk $(<5 \%)$, moderate risk (5-9.9\%), high risk (10-14.9\%) and very high risk (>15\%) [15].

As parameters of kidney function, we used the estimated glomerular filtration rate (eGFR) and the urine albumin-to-creatinine ratio (UACR). The Chronic Kidney Disease Epidemiology Collaboration formula [16] was used to estimate eGFR based on serum creatinine levels obtained after overnight-fasting conditions in all patients. Patients were dichotomized using the cut-off value of $60 \mathrm{ml} / \mathrm{min} / 1.73 \mathrm{~m}^{2}$ to classify those with chronic kidney disease (CKD). To avoid the inclusion of end-stage renal disease, one subject was excluded for the present analysis because eGFR was $<15 \mathrm{ml} / \mathrm{min} / 1.73 \mathrm{~m}^{2}$. UACR was assessed in a single-spot urine sample collected on the same day of the baseline visit and sent to central laboratory for determination. As UACR was not normally distributed in our population, the natural log transformation of this variable was used for the analysis as a continuous variable. Additionally, we performed the analysis also considering the presence or absence of microalbuminuria (defined as albumin:creatinine ratio $>21 \mathrm{mg} / \mathrm{g}$ in men and $>30$ in women) [17].

\section{Brain MRI and the Combination of CSVD Markers}

A brain MRI was performed within the next month after study entry. All examinations were obtained with the same 1.5 tesla MR (Signa HDx 1.5, General Electrics, Waukeska, Wisc., USA), including axial T1, T2 and fluid-attenuated inversion recovery (FLAIR) weighted images. Details on our data acquisition protocol have been published elsewhere [14]. We identified several markers of CSVD and applied a CSVD ordinal scale that has been detailed elsewhere $[10,13]$. Briefly, one point was awarded for the presence of each of the following 4 lesions (fig. 1): (1) $\geq 1$ lacunes 
Fig. 1. Representative images for components of the CSVD scale. At the top row are displayed FLAIR MRI, extensive periventricular and subcortical WMH (a) and a right caudate lacune (b). Extensive basal ganglia EPVS (circles in c) and one microbleed in the left striatum (arrow in $\mathbf{d}$ ) are displayed at the bottom panel.

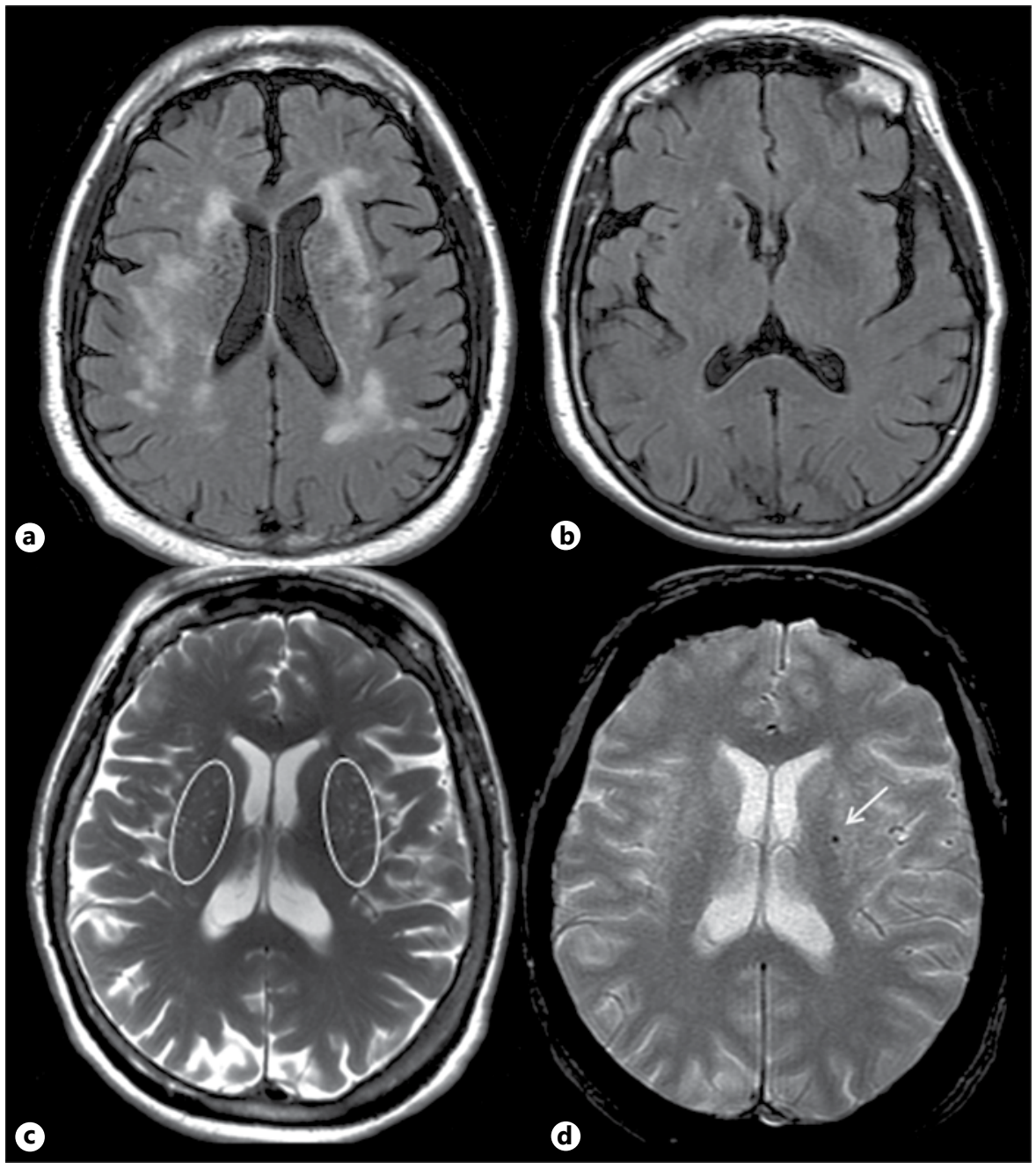

(identified as lesions with cerebrospinal fluid-like signal characteristics in all pulse sequences and with the presence of a hyperintense rim surrounding the lesion in FLAIR, located in the territory of a perforating arteriole, with a maximum diameter comprised between 3 and $15 \mathrm{~mm}$ ); (2) WMH rated at FLAIR sequences and scoring 3 points at periventricular areas or 2-3 points in the deep WM, according to the Fazekas scale [18]; (3) moderate to extensive ( $>10)$ basal ganglia EPVS assessed on T2-weighted MRI; and (4) $\geq 1$ deep brain microbleeds (rounded hypointense lesions on T2-weighted gradient echo images, with a diameter of $<10 \mathrm{~mm}$ and located in the basal ganglia, thalamus and internal or external capsules). All lesions were defined based on the STRIVE criteria [19].

All MRI were assessed by 2 neuroradiologists and an experienced stroke neurologist and disagreements were solved by consensus. Intrarater agreement was calculated for each reader in a training set before undertaking the present reading and ranged from 0.60 to 0.75 . The main source of disagreements came from the differentiation of lacunes from EPVS; therefore, lesions located in areas with high prevalence of EPVS such as the lower part of basal ganglia, were not considered lacunes.

\section{Statistical Methods}

Normality for continuous variables was assessed with the Kolmogorov-Smirnov test. Significance for intergroup differences was assessed by the $\chi^{2}$ or Fisher's exact test for categorical variables and by the Student $t$ test, analysis of variance, Mann-Whitney $U$ and Kruskal-Wallis test for continuous variables. The correlation between continuous variables was determined with Spearman's or Pearson's coefficients, as appropriate.

We studied the relationship between the presence of vascular risk factors, duration of HTN, treatment with BP-lowering drugs and kidney function (assessed by the creatine-based eGFR and the UACR) with the score on this CSVD scale in univariate analyses. Since the number of participants scoring $3(n=13)$ and $4(n=4)$ points in the scale was low, we joined them in one group (3-4 markers).

Next, we performed multivariable ordinal regression analyses considering the CSVD score as the outcome and kidney function as predictor and controlling for all covariates showing an association with the CSVD score $(\mathrm{p}<0.10)$ in the univariate analysis.

In secondary analyses, we performed the same models considering all markers comprised in the scale separately. All analyses
Vilar-Bergua et al. 
Table 1. Factors associated with the combination of markers of CSVD

\begin{tabular}{|c|c|c|c|c|}
\hline Characteristics & $\begin{array}{l}\text { No marker } \\
(\mathrm{n}=700)\end{array}$ & $\begin{array}{l}\text { One marker } \\
(\mathrm{n}=192)\end{array}$ & $\begin{array}{l}2 \text { markers } \\
(\mathrm{n}=66)\end{array}$ & $\begin{array}{l}3-4 \text { markers } \\
(\mathrm{n}=17)\end{array}$ \\
\hline Age, years & $63(58-67)$ & $65(61-68)$ & $66(63-68)$ & $66(63-70)^{* *}$ \\
\hline Gender, male, \% & 47.3 & 51 & 57.6 & $82.4^{* *}$ \\
\hline Active smoker, \% & 14.9 & 16.1 & 16.7 & 11.8 \\
\hline Alcohol abuse, \% & 6.1 & 10 & 2.6 & 0 \\
\hline Dyslipidemia, \% & 72.3 & 68.4 & 77.3 & 64.7 \\
\hline Diabetes, \% & 22.1 & 26.6 & 27.3 & 23.5 \\
\hline $\mathrm{BMI}, \mathrm{kg} / \mathrm{m}^{2}$ & $30(27.1-33.2)$ & $30.2(27.8-33.3)$ & $29.4(26.8-33.9)$ & $28.3(26.1-31.1)$ \\
\hline \multicolumn{5}{|l|}{ Global vascular risk, \% } \\
\hline Low risk & 25.5 & 24.6 & 9.2 & $5.9^{* *}$ \\
\hline Moderate risk & 46.3 & 42.9 & 40 & 47.1 \\
\hline High risk & 12.5 & 16.8 & 20 & 23.5 \\
\hline Very high risk & 15.7 & 15.7 & 30.8 & 23.5 \\
\hline Previous CV disease, \% & 11.4 & 12 & 22.7 & 5.9 \\
\hline Office SBP, mm Hg & $142(133-152)$ & $142(129-155)$ & $144(131-156)$ & $146(135-174)$ \\
\hline Office DBP, mm Hg & $78(71-84)$ & $79(71-84)$ & $79(72-84)$ & $84(73-88)$ \\
\hline HTN duration, years & $8.3(5.2-12.2)$ & $8.2(5.2-12.4)$ & $10.2(7.6-15.8)$ & $9.3(4.7-14.4)^{*}$ \\
\hline \multicolumn{5}{|l|}{ Number of BP lowering drugs, $\%$} \\
\hline None & 5.4 & 3.6 & 0 & $0^{* *}$ \\
\hline Monotherapy & 42.1 & 36.5 & 33.3 & 29.4 \\
\hline 2 or more & 52.4 & 59.9 & 66.7 & 70.6 \\
\hline SBP control, poor, $\%$ & 53.9 & 51.8 & 58.5 & 64.7 \\
\hline DBP control, poor, $\%$ & 8.3 & 11 & 12.3 & 17.6 \\
\hline Treatment adherence, $\%$ & 54.9 & 53.6 & 54.7 & 41.2 \\
\hline Ln UACR & $2.0 \pm 1.1$ & $2.2 \pm 1.2$ & $2.3 \pm 1.4$ & $2.9 \pm 1.4^{* *}$ \\
\hline $\mathrm{eGFR}, \mathrm{ml} / \mathrm{min} / 1.73 \mathrm{~m}^{2}$ & $90(78-96)$ & $88(73-96)$ & $87(75-93)$ & $87(71-93)$ \\
\hline Microalbuminuria, yes, $\%$ & 12 & 15.8 & 21 & $52.9^{* *}$ \\
\hline $\mathrm{eGFR}<60 \mathrm{ml} / \mathrm{min} / 1.73 \mathrm{~m}^{2}, \%$ & 5.3 & 10.4 & 9.8 & 6.7 \\
\hline
\end{tabular}

Variables are expressed as percentage or median (interquartile range) as appropriate.

$\mathrm{BMI}=$ Body mass index; SBP = systolic blood pressure; DBP = diastolic blood pressure; Ln UACR = natural logarithmic transformation of UACR.

${ }^{*} \mathrm{p}$ value $<0.1,{ }^{* *} \mathrm{p}$ value $<0.05$.

were performed with SPSS 17.0 statistical package (Chicago, Ill., USA). Two-tailed p values $<0.05$ were considered to indicate statistical significance.

\section{Results}

Nine hundred seventy five subjects were included in this study. The mean age was 64 (59-67) and $49.3 \%$ were men. Among them, 275 subjects $(28.2 \%)$ presented at least one CSVD marker (19.7\% one, $6.8 \%$ two, $1.3 \%$ three and $0.4 \%$ all 4 markers). From those with one marker, the most common was the presence of moderate to extensive basal ganglia EPVS (77.1\%), followed by the presence of one or more lacunes (12.5\%), WMHs (7.3\%) and deep brain microbleeds (3.1\%). Regarding combinations, any possible combination was found, being the most frequent marker included in any combination, the presence of EPVS.

\section{Clinical Characteristics Related to the Combination of CSVD Markers}

Next, we studied which clinical factors were associated with the combination of CSVD markers (table 1). We found that increasing age $(\mathrm{p}<0.001)$ and male gender $(\mathrm{p}=0.004)$ were associated with the increasing number of different CSVD markers. No associations were found between any individual cardiovascular risk factor (i.e. diabetes, dyslipidaemia, smoking) and the combination of CSVD markers; however, the estimated CV risk assessed by the REGICOR score was significantly associated with increasing CSVD scores $(\mathrm{p}<0.001)$. Almost all partici- 
Table 2. Associations of UACR and eGFR with CSVD

\begin{tabular}{|c|c|c|c|}
\hline Kidney function parameters & \multicolumn{3}{|l|}{ Score of CSVD, OR (95\% CI) } \\
\hline Microalbuminuria (yes/no) & $2.02(1.37-2.97), \mathrm{p}<0.001$ & $2.19(1.40-3.42), \mathrm{p}<0.001$ & $2.40(1.44-3.98), \mathrm{p}=0.001$ \\
\hline $\mathrm{eGFR}, \mathrm{ml} / \mathrm{min} / 1.73 \mathrm{~m}^{2}$ & $0.99(0.98-1.01), p=0.37$ & $0.99(0.98-1.01), p=0.30$ & - \\
\hline eGFR ( $\leq 60$ vs. $>60), \mathrm{ml} / \mathrm{min} / 1.73 \mathrm{~m}^{2}$ & $1.64(0.91-2.98), \mathrm{p}=0.10$ & $1.52(0.78-2.96), \mathrm{p}=0.22$ & - \\
\hline
\end{tabular}

Ln UACR = Natural logarithmic transformation of UACR.

Model 1 is adjusted by age and gender; model 2 is additionally adjusted by: CV risk (REGICOR) score, hypertension duration, number of BP-lowering drugs and diastolic BP control; model 3 is additionally adjusted by eGFR. REGICOR score variable was used in the logistic regression model taking the first category (low risk) as reference.

pants in this cohort (95.4\%) were treated with BP-lowering drugs; a significant and positive relationship was found between the number of BP-lowering drugs taken by the patient and the score of CSVD $(\mathrm{p}=0.001)$. Also those with longer duration of HTN $(p<0.05)$ and poorer diastolic BP control $(\mathrm{p}=0.06)$ were more represented at the highest scores as compared to those well controlled or more recently diagnosed.

\section{Kidney Function and the Combination of CSVD \\ Markers}

Median eGFR in this sample was $88.6 \mathrm{ml} / \mathrm{min} / 1.73 \mathrm{~m}^{2}$ and $6.6 \%$ of subjects were classified as having CKD. Regarding the UACR, $13.5 \%$ had microalbuminuria. We found increasing proportions of subjects with microalbuminuria (or higher log transformed UACR) with increasing number of markers of CSVD $(p<0.001$ and $p=0.004$, respectively).

Considering the patients with CKD (eGFR $<60 \mathrm{ml} /$ $\min / 1.73 \mathrm{~m}^{2}$ ), they were more represented in those with at least 1 point than in those with zero markers $(\mathrm{p}=0.08)$, but a linear decrease in eGFR at increasing CSVD scores was not found $(\mathrm{p}=0.15)$.

In order to assess whether these kidney function parameters were related with the combination of CSVD markers independently of age, gender and vascular risk factors or BP-lowering treatments, we performed multivariate ordinal regression analyses and results are presented in table 2. Only the UACR (or microalbuminuria) was independently associated with the score of CSVD, whereas no significant associations were found regarding eGFR. Microalbuminuria (but not eGFR) remained as an independent predictor for the markers assessed with the CSVD scale considered separately, except for the presence of deep micro- bleeds that were not associated (online suppl. material; for all online suppl. material, see www.karger.com/ doi/10.1159/000445168).

\section{Discussion}

In this study, we showed that a significant proportion of asymptomatic hypertensive subjects (almost 30\%) present at least one marker of CSVD, the presence of moderate to extensive EPVS, being the most frequent of them (alone or in combination). Furthermore, we showed that the UACR was independently associated with the presence of an increasing number of different CSVD markers, whereas kidney function measured by the creatinine-estimated GFR was not.

To our knowledge, only one prior study has investigated the relationship between different parameters of kidney function and the combination of imaging markers of CSVD [13]. They applied the same scale to a cohort of subjects with first-ever lacunar stroke and found that both proteinuria and low eGFR were associated with the co-occurrence of CSVD markers. Some differences between our study and Liao's cohort should be noticed besides the study population (asymptomatic hypertensive cohort vs. first-ever lacunar stroke). In particular, our proportion of subjects scoring 3 or 4 points in the CSVD scale was smaller than in Liao's and collaborators study. Certainly, this fact might have limited our statistical power to show associations with eGFR. However, previous studies have failed to show a relationship between mild forms of CKD (considering eGFR between 60 and $90 \mathrm{ml} /$ $\mathrm{min} / 1.73 \mathrm{~m}^{2}$ ) and CSVD markers as $\mathrm{WMH}$, whereas a clear association was present in subjects with moderate to severe forms of CKD $\left(<60 \mathrm{ml} / \mathrm{min} / 1.73 \mathrm{~m}^{2}\right)$. This sug- 
gests that the relationship between eGFR and CSVD is not as clear in the context of mild CKD as for moderate to severe CKD [5]. On the contrary, associations were still present for the UACR or microalbuminuria, which was more prevalent in our sample.

The association between individual markers of CSVD (i.e. lacunes, $\mathrm{WMH}$ and cerebral microbleeds) and kidney function has been extensively studied before [3-9, 20]. We found that associations with microalbuminuria were present for lacunes, WMH and basal ganglia EPVS (the most frequent marker in our study). Based on our results, WMH and basal ganglia EPVS were the most strongly associated markers in relation with microalbuminuria.

Since the vasculature of brain and kidney display similar anatomical and hemodynamic characteristics [21] (i.e. both are exposed to high pressure fluctuations and low resistance to flow), vascular disease in both organs is thought to represent different manifestations of a systemic small vessel disease, associated with shared risk factors, inflammation and endothelial dysfunction, among other mechanisms [22]. The discrepant associations between CSVD markers and microalbuminuria or eGFR might be related to the fact that microalbuminuria is a marker of glomerular endothelial dysfunction that might be occurring simultaneously in the brain in the context of a CSVD.

In our study, almost $30 \%$ of subjects presented at least 1 CSVD marker; this proportion is lower than in a previous report from hypertensives (39.3\%) [11] and largely different from a lacunar stroke cohort (85\%) [10]. However, consistently with those reports, EPVS were the most prevalent lesion. Some authors have suggested that EPVS may appear early in the course of CSVD [23] and this would explain their relatively higher prevalence as compared to other markers of CSVD. Future prospective studies with longitudinal MRI assessment would be needed to confirm these assumptions.

Our study has both strengths and limitations. As strength, it has been performed in a large cohort of asymptomatic hypertensives recruited randomly from a general population, avoiding selection biases in more specialized units. We provided associations between a global assessment of CSVD and kidney function (considering possible linear associations and clinically defined thresholds) and also considering the markers separately.

Also we have limitations; although the scale of CSVD applied in this study combines the presence of different imaging markers, it does not take into account the actual burden of each marker and gives an equal weightage to all of them. Although the clinical consequences of the individual markers comprised in the scale may be rather different, overall, the scores are directly associated with ambulatory blood pressure measurements [10] and inversely correlated with the cognitive function [11]. This might be clinically relevant.

Finally, microalbuminuria was measured in this study in a single-spot urine sample, and not confirmed with multiple testing, although we provided a quantitative measurement relative to urine creatinine.

\section{Conclusions}

In this study, we showed that almost $30 \%$ of asymptomatic hypertensive individuals present at least one marker of CSVD. Furthermore, our data suggest that microalbuminuria may constitute a surrogate marker for the combination of markers of CSVD. This observation might be clinically relevant if we consider that CSVD is a condition characterized not only by the accumulation of imaging markers but by the occurrence of progressive vascular cognitive impairment and stroke. Future studies with simultaneous assessment of UACR and CSVD over time are warranted.

\section{Acknowledgements}

This research has been funded by Instituto de Salud Carlos III (grants PI10/0705 and PI14/1535, ICI14/00307) co-financed by the European Regional Development Fund (FEDER), the Spanish Ministry of Health (EC10/336), the Catalonian Society of Hypertension (6th grant research on hypertension) and the Càtedra UAB-Novartis de Medicina de familia. Neurovascular Research Laboratory receives funding from URIACH and takes part in the Spanish stroke research network INVICTUS (RD12/0014/0005).

\section{Sources of Support}

A. Vilar-Bergua is funded by a predoctoral contract from the Fundació Josep Palau Francàs and Vall d'Hebron Research Institute. I. Riba-Llena was the recipient of a research contract from the Spanish Ministry of Health (CM10/00063). P. Delgado is partially supported by the Miguel Servet program (CP09/136) from the Instituto de Salud Carlos III.

\section{Disclosure Statement}

None to declare. 


\section{References}

1 Pantoni L: Cerebral small vessel disease: from pathogenesis and clinical characteristics to therapeutic challenges. Lancet Neurol 2010;9: 689-701.

2 Wardlaw JM, Smith C, Dichgans M: Mechanisms of sporadic cerebral small vessel disease: insights from neuroimaging. Lancet Neurol 2013;12:483-497.

3 Akoudad S, Sedaghat S, Hofman A, Koudstaal PJ, van der Lugt A, Ikram MA, Vernooij MW: Kidney function and cerebral small vessel disease in the general population. Int J Stroke 2015;10:603-608.

4 Otani H, Kikuya M, Hara A, Terata S, Ohkubo T, Kondo T, Hirose T, Obara T, Metoki H, Inoue R, Asayama K, Kanno A, Terawaki H, Nakayama M, Totsune K, Hoshi H, Satoh H, Izumi S, Imai Y: Association of kidney dysfunction with silent lacunar infarcts and white matter hyperintensity in the general population: the Ohasama study. Cerebrovasc Dis 2010;30:43-50.

5 Khatri M, Wright CB, Nickolas TL, Yoshita M, Paik MC, Kranwinkel G, Sacco RL, DeCarli C: Chronic kidney disease is associated with white matter hyperintensity volume: the northern Manhattan study (NOMAS). Stroke 2007;38:3121-3126.

6 Wada M, Nagasawa H, Kawanami T, Kurita K, Daimon M, Kubota I, Kayama T, Kato T: Cystatin $\mathrm{C}$ as an index of cerebral small vessel disease: results of a cross-sectional study in community-based Japanese elderly. Eur J Neurol 2010;17:383-390.

7 Umemura T, Kawamura T, Sakakibara T, Mashita S, Hotta N, Sobue G: Microalbuminuria is independently associated with deep or infratentorial brain microbleeds in hypertensive adults. Am J Hypertens 2012;25:430436.

8 Nagai M, Hoshide S, Takahashi M, Shimpo M, Kario K: Sleep duration, kidney function, and their effects on cerebral small vessel disease in elderly hypertensive patients. Am J Hypertens 2015;28:884-893.

9 Ravera M, Ratto E, Vettoretti S, Viazzi F, Leoncini G, Parodi D, Tomolillo C, Del Sette M, Maviglio N, Deferrari G, Pontremoli R: Microalbuminuria and subclinical cerebrovascular damage in essential hypertension. J Nephrol 2002;15:519-524.

10 Klarenbeek P, van Oostenbrugge RJ, Rouhl RP, Knottnerus IL, Staals J: Ambulatory blood pressure in patients with lacunar stroke: association with total MRI burden of cerebral small vessel disease. Stroke 2013;44:29952999.
11 Huijts M, Duits A, van Oostenbrugge RJ, Kroon AA, de Leeuw PW, Staals J: Accumulation of MRI markers of cerebral small vessel disease is associated with decreased cognitive function. A study in first-ever lacunar stroke and hypertensive patients. Front Aging Neurosci 2013;5:72.

12 Staals J, Makin SD, Doubal FN, Dennis MS, Wardlaw JM: Stroke subtype, vascular risk factors, and total MRI brain small-vessel disease burden. Neurology 2014;83:1228-1234.

13 Xiao L, Lan W, Sun W, Dai Q, Xiong Y, Li L, Zhou Y, Zheng P, Fan W, Ma N, Guo Z, Chen X, Xie X, Xu L, Zhu W, Xu G, Liu X: Chronic kidney disease in patients with lacunar stroke: association with enlarged perivascular spaces and total magnetic resonance imaging burden of cerebral small vessel disease. Stroke 2015; 46:2081-2086.

14 Riba-Llena I, Jarca CI, Mundet X, Tovar JL, Orfila F, López-Rueda A, Nafría C, Fernández JL, Castañé X, Domingo M, Alvarez-Sabín J, Fernández-Cortiñas I, Maisterra O, Montaner J, Delgado P: Investigating silent strokes in hypertensives: a magnetic resonance imaging study (ISSYS): rationale and protocol design. BMC Neurol 2013;13:130.

15 Marrugat J, Subirana I, Ramos R, Vila J, Marín-Ibañez A, Guembe MJ, Rigo F, Tormo Díaz MJ, Moreno-Iribas C, Cabré JJ, Segura A, Baena-Díez JM, de la Cámara AG, Lapetra J, Grau M, Quesada M, Medrano MJ, González Diego P, Frontera G, Gavrila D, Aicua EA, Basora J, García JM, García-Lareo M, Gutierrez JA, Mayoral E, Sala J, D’Agostino R, Elosua R: Derivation and validation of a set of 10 -year cardiovascular risk predictive functions in Spain: the FRESCO study. Prev Med 2014;61: 66-74.

16 Levey AS, Stevens LA, Schmid CH, Zhang YL, Castro AF 3rd, Feldman HI, Kusek JW, Eggers P, Van Lente F, Greene T, Coresh J: A new equation to estimate glomerular filtration rate. Ann Intern Med 2009;150:604-612.

17 Mancia G, De Backer G, Dominiczak A, Cifkova R, Fagard R, Germano G, Grassi G, Heagerty AM, Kjeldsen SE, Laurent S, Narkiewicz K, Ruilope L, Rynkiewicz A, Schmieder RE, Struijker Boudier HA, Zanchetti A, Vahanian A, Camm J, De Caterina R, Dean V, Dickstein K, Filippatos G, Funck-Brentano C, Hellemans I, Kristensen SD, McGregor K, Sechtem U, Silber S, Tendera M, Widimsky P, Zamorano JL, Kjeldsen SE, Erdine S, Narkiewicz K, Kiowski W, Agabiti-Rosei E, Ambrosioni E, Cifkova R, Dominiczak A, Fagard R, Heagerty AM, Laurent S, Lindholm LH, Mancia G,
Manolis A, Nilsson PM, Redon J, Schmieder RE, Struijker-Boudier HA, Viigimaa M, Filippatos G, Adamopoulos S, Agabiti-Rosei E, Ambrosioni E, Bertomeu V, Clement D, Erdine S, Farsang C, Gaita D, Kiowski W, Lip G, Mallion JM, Manolis AJ, Nilsson PM, O’Brien E, Ponikowski P, Redon J, Ruschitzka F, Tamargo J, van Zwieten P, Viigimaa M, Waeber B, Williams B, Zamorano JL; The Task Force for the Management of Arterial Hypertension of the European Society of Hypertension, The Task Force for the Management of Arterial Hypertension of the European Society of Cardiology: 2007 guidelines for the management of arterial hypertension: the task force for the management of arterial hypertension of the European society of hypertension (ESH) and of the European society of cardiology (ESC). Eur Heart J 2007;28:1462-1536.

18 Fazekas F, Kleinert R, Offenbacher $H$, Schmidt R, Kleinert G, Payer F, Radner H, Lechner H: Pathologic correlates of incidental MRI white matter signal hyperintensities. Neurology 1993;43:1683-1689.

19 Wardlaw JM, Smith EE, Biessels GJ, Cordonnier C, Fazekas F, Frayne R, Lindley RI, O’Brien JT, Barkhof F, Benavente OR, Black SE, Brayne C, Breteler M, Chabriat H, Decarli C, de Leeuw FE, Doubal F, Duering M, Fox NC, Greenberg S, Hachinski V, Kilimann I, Mok V, Oostenbrugge RV, Pantoni L, Speck O, Stephan BC, Teipel S, Viswanathan A, Werring D, Chen C, Smith C, van Buchem M, Norrving B, Gorelick PB, Dichgans M: Neuroimaging standards for research into small vessel disease and its contribution to ageing and neurodegeneration. Lancet Neurol 2013;12:822-838.

20 Makin SD, Cook FA, Dennis MS, Wardlaw JM: Cerebral small vessel disease and renal function: systematic review and meta-analysis. Cerebrovasc Dis 2015;39:39-52.

21 O'Rourke MF, Safar ME: Relationship between aortic stiffening and microvascular disease in brain and kidney: cause and logic of therapy. Hypertension 2005;46:200-204.

22 Tsioufis C, Dimitriadis K, Andrikou E, Thomopoulos C, Tsiachris D, Stefanadi E, Mihas C, Miliou A, Papademetriou V, Stefanadis $C$ : ADMA, C-reactive protein, and albuminuria in untreated essential hypertension: a cross-sectional study. Am J Kidney Dis 2010;55:1050-1059.

23 Deramecourt V, Slade JY, Oakley AE, Perry RH, Ince PG, Maurage CA, Kalaria RN: Staging and natural history of cerebrovascular pathology in dementia. Neurology 2012;78: 1043-1050. 